\title{
Appetite for Disruption: Designing Human-Centred Augmentations to an Online Food Ordering Platform
}

\author{
Louis Goffe \\ Open Lab \\ Newcastle upon Tyne, UK \\ louis.goffe@newcastle.ac.uk
}

\author{
Simon Bowen \\ Open Lab \\ Newcastle upon Tyne, UK \\ simon.bowen@newcastle.ac.uk
}

\author{
Shruthi Sai Chivukula \\ Purdue University \\ West Lafayette, Indiana, US \\ cshruthi@purdue.edu
}

\author{
Austin L. Toombs \\ Purdue University \\ West Lafayette, Indiana, US \\ toombsa@purdue.edu
}

\author{
Alex Bowyer \\ Open Lab \\ Newcastle upon Tyne, UK \\ a.bowyer2@newcastle.ac.uk
}

Colin M. Gray

Purdue University

West Lafayette, Indiana, US

gray42@purdue.edu

\begin{abstract}
Online food ordering platforms have changed how many of us purchase takeaway food. They have centralised and streamlined access, providing an opportunity for population-level dietary impact. However, they are currently not human-centred: typically providing limited functionality in support of users' values and dietary considerations; and focused on the provision of food that is broadly characterised as unhealthy. In this paper we explore a redesign of portions of Just Eat, an online takeaway food aggregator, building upon theoretical perspectives from public health. We conducted workshops in 2018 and 2019 to identify user behaviours and motivations then designed a human-centric web augmentation template that could disrupt platform provider behaviours and increase functionality to support users' desires and well-being. We provide a template for lightweight end-user appropriations of food ordering platforms that would enable researchers to explore how health information features could improve individual health and satisfaction, and design guidance for disruptively augmenting existing food ordering platforms (or designing new ones) to enable transparency, personalisation, and self-monitoring to empower users and improve their well-being.
\end{abstract}

Digital economy; Human-centred Design; UX design; Food; Health; Design practices; Web augmentation; Service design

\section{INTRODUCTION}

The UK is a nation of hot fast food takeaway lovers. The home delivery and takeaway market was valued at $£ 7.9$ billion in 2017 (Passport, 2018). Meals from independent traders often provide portions that are excessively large (Jaworowska et al., 2014), and have been linked to significant public health implications including greater body mass index and greater odds of obesity (Burgoine et al., 2014). Online food ordering platforms have grown rapidly in the past decade, with many chain restaurants developing their own websites and apps, such as Domino's and Pizza Hut, as well as aggregator websites such as Just Eat and Grubhub which provide users with the ability to order from a wide (predominately independent) variety of local takeaway food outlets. There has been further adoption of online food ordering platforms as a result of the COVID-19 pandemic (when takeaway food has been the only trading option for restaurants), e.g.
8-9\% UK growth in first two months (Shakespeare, 2020) with similar growth worldwide (Watanabe, Omori and others, 2020; McCabe and Erdem, 2021). Just Eat, Uber Eats, Deliveroo, Grubhub, and Doordash each have millions of users, but there is currently little research that describes their role in informing our dietary choices. To date, interventions that aim to support healthier choices have been targeted at activities and practices within the food outlet (Hillier-Brown et al., 2017) and often focused on calorie labelling (Bleich et al., 2017). These interventions are particularly challenging to implement, resource intensive, and require the compliance and engagement with the outlet owner and/or manager (Goffe et al., 2018). Due to the growing number of active users of online food ordering platforms, there is potential for populationlevel diet (Public Health England, 2020)and subsequent health impact. These platforms are innately user-friendly (Parker, Van Alstyne and 
Choudary, 2016). However this 'use' relates to enabling users to easily select and purchase takeaway food. From a human-centred design perspective, where consideration is given to how well these platforms support well-being and human flourishing (Buchanan, 2001), these platforms are lacking. Thus, there is an opportunity to go beyond consideration of usability (with narrow definitions of 'use') to investigate how food ordering platforms can better support well-being-related factors that influence our food choice, such as health, weight control, natural content, and ethical considerations (Scheibehenne, Miesler and Todd, 2007). A YouGov survey found that $28 \%$ of Britons who increased their takeaway usage during the pandemic are still motivated by healthy menu options and freshness of ingredients; the pandemic has not lessened the importance of supporting healthy takeaway selection.

Responding to this disconnect between user-friendly and human-centred concerns, we conducted a sequence of design research activities in 2018 and 2019 to investigate how individual goals for food ordering, including the facilitation of users' informed decision-making regarding healthy lifestyle choices, can be better supported. In framing these activities, we foregrounded health in an a priori manner, with the emergent views and findings on how to shape food ordering platforms grounded in our participants' experiences. This research had three linked objectives. The first was to understand users' processes and experiences of food ordering, which we explored using an interactive workshop in which participants detailed their thoughts, feelings, and actions during food ordering and the role of nutritional information in this process. The second objective was to co-design proposals for idealised and human-centred food ordering services, which we explored in a separate workshop where participants drew upon personal experiences through supported ideation. Our third objective was to produce a technologically feasible design that embodied the idealised feature sets imagined by our participants and researchers. To pursue this third objective, we used an agile UX framework to produce a human-centred web augmentation template for the disruption of the Just Eat platform.

Our work provides an accessible illustration of how online food ordering platforms could improve their structure to support healthier choices and other human-centred considerations by providing better tools to assess food healthiness and outlet hygiene at the point of food outlet selection. We offer a design provocation in the form of a web augmentation template for how an existing platform could support users' values and signpost them to healthier options. Furthermore, we illustrate how the proposed augmentations could impact users' task flow. In doing so, we identify fundamental design aspects of platform design, stemming from opacity.
In conclusion, we provide three design features that designers, researchers, and advocates for humancentred design can apply to other e-commerce sites to empower users and provide them with increased control over their platform use.

\section{BACKGROUND}

\subsection{Takeaway Food and Health}

More than a fifth of UK residents order a takeaway meal at least once per week, with peak consumption in those aged 19-29 years old (Adams et al., 2015). Frequent takeaway consumption is linked to an increased mean daily energy intake (Goffe et al., 2017) and it has been hypothesised that takeaway food's high energy density can override our appetite control systems and trigger over-consumption (Prentice and Jebb, 2003). To date, observational studies have focused on the geographical pattern of takeaways (Fraser et al., 2010), where there is a positive association between takeaway outlet density and increasing level of area deprivation (Public Health England, 2018). This has led some to conclude that 'the frequency and types of takeaway foods consumed by socio-economically disadvantaged groups may contribute to inequalities in overweight or obesity and to chronic disease' (Miura, Giskes and Turrell, 2012). The Internet has created the ideal landscape for digital platforms to flourish and become the dominant business model, where the value is in the network of producers and consumers (Parker, Van Alstyne and Choudary, 2016). The COVID-19 pandemic has further mediated the mass transition to digital technologies, inclusive of food, both groceries (Grashuis, Skevas and Segovia, 2020) and hot fast food takeaways (Bradshaw, 2021).

\subsection{Just Eat}

Among takeaway platforms in the UK, Just Eat has the highest number of customers and takeaway outlets. It provides a website enabling users to order from thousands of (primarily independent) hot fast food takeaway outlets across the country. In 2018, in the UK they processed 122.8 million orders from 12.2 million active users (a respective $17.0 \%$ and $16.2 \%$ increase from 2017) (Just Eat plc, 2019d). It is not known if such platforms are increasing our consumption of takeaway food, or simply providing an alternative method of ordering. What is known, from their annual accounts (Just Eat plc, 2019d), is that Just Eat is hugely popular as it eases access to takeaway food and for many is likely to be the primary means to acquire such food.

As a public limited company, Just Eat is motivated to maximise profits. The majority of its revenue is generated from a commission of $14 \%$ plus tax charged to each food outlet on every order 
processed (Just Eat plc, 2020). Therefore, increasing orders from all outlets is their priority, and issues relating to the public's health do not factor unless there is negative media reporting that has the potential to impact on their revenue. The prominence of media reporting suggests that the results of an independent food safety inspection would be of interest to users to assure them that the food they are purchasing is safe to eat, but Just Eat does not present this information prominently to users. Furthermore, an investigation by the BBC found that Just Eat were routinely listing without warning takeaways that had received a food safety score of zero (Crawford, 2018), which means that urgent improvement is necessary to prevent a risk to public health (Food Standards Agency, 2018). Just Eat announced in July 2019 that it would display the hygiene rating for all businesses listed on its platform (Anon, 2019), however, this is not offered at the point of takeaway outlet selection and requires prior knowledge by the user to locate the rating. This highlights the conflict between Just Eat's stated desire to 'give our customers an amazing experience' (Just Eat plc, 2019c) and the primacy of their profit motive (Just Eat plc, 2019a).

Just Eat presents an appearance of impartiality and places the emphasis on their users' reviews to determine outlet quality. They have carefully balanced the appearance and functionality of the platform with regards to both takeaway owners and consumers, where the primary outcome of interest is profit. As the end users are largely unaware of these design constraints, there is minimal advocacy for improved functionality and general contentment with what the service provides. To avoid loss of profit, platform development would likely focus on features related to usability such as ease of purchasing and neglect validated measures that are critical of specific takeaway outlets.

As independent researchers absent of responsibility to provide profit to Just Eat shareholders, we can take a human-centred approach to this sociotechnical design challenge of going beyond the user friendliness of the takeaway transaction to support human well-being. We view the popularity of online food ordering platforms as an opportunity to identify how platforms might support healthy food choices as for many they may become their primary means to access a hot meal.

\subsection{Web Augmentation for Design After Design}

The desire to use products in ways that have not been anticipated can be explored through the practice of Adversarial Design (DiSalvo, 2012), whereby design processes are used to challenge the status quo. Storni identified the idea of 'empowerment-in-use', which advocates 'design after design', an application of traditional design techniques to the problem of how users might appropriate their existing technology to different uses that might not have been foreseen by the designers (Storni, 2014). Applying this philosophy, we can consider that a digital platform is simply a product that, like any other, a user might wish to adapt to better suit their needs. When considering how a product might be adapted, it is important to consider the 'seams'-those exposed areas that the user is free to change (Maccoll and Chalmers, 2003). Technology providers often remove seams, reducing the possibility of design after designensuring users behave more uniformly, often to limit maintenance costs. Examples of removing seams include manufacturers such as Apple or Samsung making it difficult for users to open their own phones (e.g., to replace batteries), Facebook removing RSS feeds, or Twitter closing its APIs.

When a website is viewed, the loaded web page then exists on the user's local device within the web browser at the point of interaction-creating a seam that cannot be removed and where the service provider's power to influence the user's interaction is reduced. This opportunity is exploited by the mechanism of web augmentation (Díaz, 2012; Díaz, Arellano and Azanza, 2013; Díaz et al., 2014; Díaz and Arellano, 2015), in which a user's experience is modified using a browser extension or plugin to manipulate the loaded web page in order to remove, add, or modify elements of the page before the user interacts with it. Well-known examples are adblockers that remove unwanted banners, advertisements, or pop-ups from pages. In research, web augmentation has been used to stop clickbait (Chakraborty et al., 2016), filter explicit words (Suliman and Mammi, 2017), dispute fake news (Ennals, Trushkowsky and Agosta, 2010), and to combat addiction (Pyshkin et al., 2016).

Given the evidence linking exposure and consumption of takeaway food and the rapid growth of online food ordering platforms, Just Eat provides an ideal context to design web augmentations that make a platform more human-centred and support users in making choices that align to their personal values and dietary requirements.

\section{RESEARCH DESIGN}

Our research consisted of three sequential stages involving design activities to explore current experiences of-and design possibilities fortakeaway food ordering with consumers of such food. Stage one sought to understand participants' experiences of food ordering through a workshop. Stage two sought to generate novel, human-centred design proposals for food ordering services through a co-design workshop. Stage three synthesised findings from stages one and two into a design consisting of proposals for the web augmentation of 
Just Eat to empower its users, subject to the known capabilities and limitations of web augmentation. Hence, the findings from stages 1 and 2 are summarised alongside their descriptions below. Ethical approval for this study was provided by Newcastle University ethics committee (Reference: 5377/2018). All participants gave written informed consent to take part in the study.

\subsection{Stage 1 (S1): Experiences of Ordering Takeaway Food}

\subsubsection{S1 Setting and Participants}

We ran a 90-minute workshop in a Newcastle University (NU) catering outlet that was accessible to the general public, thereby immersing participants in the subject matter. Our objective was to understand and detail participants' experience and processes of ordering takeaway food. We recruited 17 participants through promotional material placed in University catering outlets and academic mailing lists. This was an appropriate target audience as young adults aged 19-29 are the most frequent consumers of takeaway food (Adams et al., 2015). Participants included: six nutrition researchers, four $\mathrm{HCl}$ researchers, and seven regular users of University catering services (staff and students from unrelated fields). Participants were split into four groups and guided through the workshop by five facilitators-two of the authors and three userexperience (UX) design students. All participants were given a £20 online shopping voucher.

\subsubsection{S1 Activities}

The workshop comprised three key activities designed to deconstruct and describe the processes and experiences of food ordering. In the first activity (30 minutes), the participants recounted to their group members a personal recent experience of ordering food in either a restaurant, café, or takeaway. They were asked to consider the various steps in that journey, including what they were thinking before and when they received the food. Each group selected one experience and created a chronological user journey map (Tomitsch et al., 2018) where they detailed each step with regards to what they were thinking, feeling, and doing. For the second activity (15 minutes), we investigated the influence of nutritional information upon participants' meal choices. Participants were asked to select and explain their most and least preferred meal from a selection of three based on an image of the food and a brief description. Following their choice, participants were presented with the following nutritional information for each meal: energy (kcal), protein $(\mathrm{g})$, fat $(\mathrm{g})$, and carbohydrates $(\mathrm{g})$. They were then asked if they would like to revise their first choice and, if so, to state their reason for changing. In the final activity (30 minutes), each group returned to their journey map and detailed at what stage the provision of specific pieces of information, such as ingredients, nutrition, allergens, price, and food outlet reputation, would be of use during the food ordering process.

\subsubsection{S1 Findings}

The user journey maps included a range of emotions about ordering takeaway food. Many participants considered that takeaway food was broadly a 'treat', and that taste, as opposed to health, was their primary consideration. However, after having eaten takeaway food, they also expressed feelings of regret and a desire for nutritional information to inform their choice. Some participants changed their meal choice to one they perceived as healthier upon provision of calories and other nutritional measures.

The user journey maps consisted of three phases: pre-order outlet decision, order, and post-order reflection. The pre-order phase consisted of feelings of hunger, or those that were a direct result of their hunger, for example 'hangry' [hunger-angry]. This triggered thoughts related to the logistical issues of identifying a suitable food outlet for the individual or party who want to eat. The order phase was the point of direct engagement with the selected food outlet. This was associated with feelings that included confusion, relief, excitement, and impatience. In the post-order phase, participants considered if they were satisfied with their food and outlet choice. Here they listed feelings related to satiation, but also reflective emotions such as regret. Provision of nutritional information motivated four of the 17 participants subsequently changed their meal choice to one they considered healthier. Other participants had their choice affirmed as the nutritional information corroborated their a priori nutritional knowledge. As with the user journey maps, the desired point of information provision was split between the three respective phases. During pre-order, users wanted information on the type of cuisine, dietary requirements, rating and reviews, cost, and opening hours. At the point of ordering, users wanted information regarding the cost of specific dishes, distance to outlet, outlet access, ingredients, meal choice of other individuals in their party, and delivery time. Upon meal reflection, users stated a desire for nutritional information.

The ordering, particularly of takeaway food, was instinctive and driven by hunger and a craving for a particular type of food. Environmental factors, including the social setting, were key determinants of what was ordered. Most participants positioned such food as a treat, inferring infrequent consumption, thus placing greater weight on their enjoyment of such food over health considerations.

Health was not a consideration in the pre-order phase. However, during the post-order phase, once participants had had the chance to reflect, some reported feelings of regret and resentment over their food choices. Broadly, the healthiness of the food 
was not considered when purchasing takeaway food. Some participants reassessed their choices when forced to consider specific nutritional information within a controlled setting, opting for a healthier choice as judged by their interpretation of the presented information, suggesting such information is desirable to inform their meal choice.

\subsection{Stage 2 (S2): Novel Design Proposals for Online Food Services}

\subsubsection{S2 Setting and Participants}

A second workshop (two hours) was conducted close to a University catering outlet used by participants. The objective of this workshop was to develop idealised, human-centred design concepts for takeaway food ordering. We recruited 16 participants, including eight participants from S1, with additional recruitment through promotional material placed in University catering outlets and academic mailing lists. As with S1, participants were deemed to be an appropriate target audience (Adams et al., 2015)(Adams <i>et al.</i>, 2015) and consisted of: six nutrition researchers, seven $\mathrm{HCl}$ researchers, and three regular users of University catering services (staff and students from unrelated fields). Participants were split into four equal groups and subsequently merged into two groups for the final activity and guided through the workshop by three facilitators. A $£ 20$ online shopping voucher was offered in recognition of participation.

\subsubsection{S2 Activities}

This workshop consisted of three phases. Firstly (30 minutes), a critique of a current takeaway food service. Participants were provided with a £5 meal voucher for a NU food outlet and asked to purchase a meal that they considered healthy, but otherwise had autonomy regarding their choice. To initiate critical thinking, we asked them what they bought, why, and how they made that choice. Additionally, we asked them to mentally note what information they looked or asked for, found, or felt was missing. Within their groups, they ate their food together and discussed and detailed the positive ('gains') and negative ('pains') aspects of their ordering experience. Having drawn attention to factors that influence food choice, we moved to the second phase (30 minutes) of supported ideation where each group generated a series of novel food ordering service ideas based on ideation cards which covered the topics: who is this idea for; what is the scenario of use; what food service is being used; what are the user's requirements; and a free-choice 'wildcard' (which included futuristic/fictional technologies such as robots, A.I. or teleportation). In the final phase (50 minutes), the four groups were combined into two equal groups of eight. Here, participants filtered, refined, and combined ideas into one conceptual idealised service, per group, for potential implementation.

\subsubsection{S2 Findings}

An emergent theme from the ideated food ordering services was the ability of the user to easily identify food that matches one's personal values and dietary preferences. Such ideas were predicated on the availability of data such as ingredients, nutritional information, and allergens. Building upon this, tools that would enable users to monitor and regulate consumption were desirable. Other ideas expressed and developed related to strong feelings regarding the social aspect of food and communal dining.

Clustering into themes the positive and negative aspects of their food ordering experience revealed that participants liked: the availability of made-toorder food; deli customisation; quick and convenient service; availability of healthy options; friendly staff; good value food; and environmentally conscious operation. Limitations of the food outlet were: limited ingredient and nutrition information; poor outlet layout; long time to pay; potential for cross-contamination of deli options; poor complete meal deals; and restricted cuisine diversity.

The groups produced 16 novel service ideas, including preferred service options for: a robot meal delivery service; the monitoring of body functioning in relation to food and outlet experience; an exercise-consumption calorie trading platform; a teleportation service linked to smartphone location; a 'loyalty-carb' tracker to support those on a carbohydrate-restricted diet; a vegan, nutritionallycontrolled portions buffet; and an app to support communal dining that accounted for each user's nutritional requirements.

For implementation, one group proposed a model for food ordering similar to the social fitness platform, Strava (Strava, 2021). The main currency would be calories and these would link to their personal level of physical activity. Users would be able to purchase meals and gain credit through the volume of exercise in which they had engaged. The social aspect would allow users to share their exercise statistics and where they had purchased their meals to create a resource of food outlets where meals could be purchased that are conducive for endurance activities. The other group presented a platform to support facilitation of social dining. It would help users to identify and coordinate a suitable food outlet where they could meet and purchase food aligned to their specific dietary preferences with their friends. It would also aim to reduce social isolation through helping users to find a 'lunch buddy' to meet for a meal.

The provision of information was critical to help support participants in relation to their food choices. They liked to know if food aligned to their personal values and dietary requirements. Takeaway food eating was greater than the simple act of satiating hunger. The social experience was viewed as highly 
valuable, where mealtime provides an opportunity to engage with others in a non-formal setting (Dunbar, 2017). Diversity of cuisine and food component options was seen as a benefit, but participants wanted to be appropriately sign-posted through the ordering process. There was a desire for services to provide a personalised and tailored ordering experience aligned to users' ideals, built on an increased provision of information on food options. The social concepts showed the increased value that participants place upon recommendations from those within their own networks. The novel platform ideas offered the potential to establish dietary goals. With these proposed features users could track, recall, and report dietary habits, with the potential to reward health-promoting activities. Additionally, the social aspects of the platform could provide peer support for users to achieve dietary goals, for example identifying suitable food outlets that adhere to an individual's dietary preferences.

\subsection{Stage 3 (S3): Designing an Augmented Just Eat to Empower Users}

\subsubsection{S3 Setting and Participants}

In our third stage, our objective was to design a human-centred web augmentation template that would incorporate those appropriate and applicable findings from S1 and S2. Where participants stated a desire for functionality that would easily enable them to identify food that matched their personal values and dietary preferences as well as monitor and regulate consumption. We wanted to identify modifications with the potential to support improved well-being of Just Eat users. To this end, a design sprint was conducted over a week ( 5 hours a day) with a group of five UX experts and practitioners on campus at NU. The sprint was an iterative process based on the agile UX framework (Chamberlain, Sharp and Maiden, 2006), delivered by a team of one postgraduate and four undergraduate UX students from Purdue University, with experience of UX methods and qualitative research methods, and was supported by design and public health academics from Open Lab (NU) and Purdue University.

\subsubsection{S3 Activities}

The design sprint was divided into four definitive phases to capture the design elements currently absent on Just Eat that could be implemented through web augmentation tools to create a more human-centred platform. Firstly, a review of the internal reporting, findings, and workshop illustrations from S1 and S2 was carried out by the design researchers. Details about the workshops conducted and insights were leveraged by the design sprint members to ideate. Secondly, the design researchers delivered an affinity analysis (Hartson and Pyla, 2012) of 'customer reviews' and ratings from existing online food ordering platforms:
Just Eat, Deliveroo, Uber Eats (all UK-based), and Grubhub and Doordash (US-based), as well as their Google Play Store app listings; to get access to user complaints and needs. The affinity analysis of 'negative' experiences shared through the comments resulted in broad themes such as: 1) late delivery; 2) incorrect information; 3) packaging problems; 4) requests to present hygiene ratings on the website; 5) staff's disrespectful attitudes; and 6) Complaints about Payment Options. This provided user insight and identification of a range of problems and needs at both the outlet- and platform-level, and we used these insights to create a user journey map (Tomitsch et al., 2018) as shown in grey in Figure 1, to detail the existing task flow for Just Eat users. The third phase was the ideation and subsequent wireframing of webaugmentable human-centred concepts applicable to the Just Eat platform (as seen in red in Figure 1). The final phase consisted of modelling the potential impact of our proposed new features on users' task flow on Just Eat by plotting concepts on to the user journey map and running user scenarios.

\subsubsection{S3 Findings: Web Augmentations of Just Eat}

The S1 and S2 findings recognised users' diverse needs and requirements for online food ordering platforms. Whilst the novel ideas generated in S2 had specific functionality (though not all were applicable to web augmentation of Just Eat), they were all predicated on an increased availability of information on both the outlet and their food offering. Based upon this, the design sprint members developed their ideas using the existing nutritional information requirements and considered the different stages at which that information might be expected by the user. This information was paramount to allow for an ordering experience tailored to the user to help them select food items that were aligned to their personal values and dietary preferences. This human-centred approach, whilst not restricting choice, would support easier identification of healthier food. The affinity analysis revealed that, in terms of features, generic components included: location, delivery time, delivery updates, door delivery, pick-up service, and menu item listings.

For specific dietary considerations (e.g., allergens), this information was the responsibility of the food outlet and users were directed to contact the outlet directly. The user reviews on the food ordering platforms were food outlet specific. While this provided an understanding with the main themes that users liked or found frustrating about ordering food-for example timely delivery, poor customer service, and packaging problems-they provided little critical insight as to the functioning of the service offered by these platforms. Data aligned to our design goals was found in Google's Play Store. This highlighted users' frustration regarding lack of 


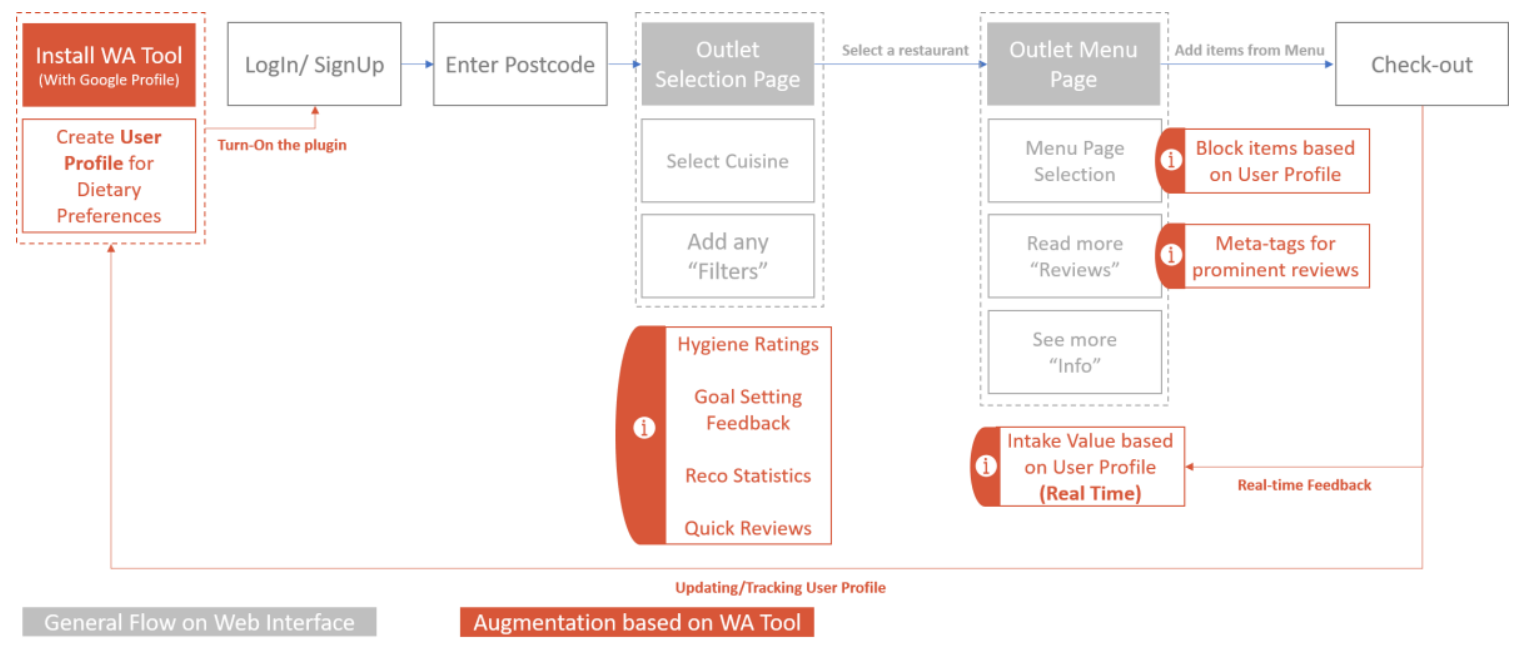

Figure 1: Just Eat user journey map with proposed augmentations in red

information from the platform on a food outlet's hygiene, ingredient and allergen information, and payment problems. The current users' journey map on the Just Eat platform is illustrated in Figure 1 in grey as 'General Flow on Web Interface'. This figure also illustrates the experience of the user after incorporating the designed feature augmentations subsequently detailed in Section 4.3. S3's ideation phase produced a design whereby new features could be augmented onto three specific parts of the current the Just Eat task flow: User Profile (which is not currently part of the Just Eat ordering flow), Outlet Selection Page, and Outlet Menu Page. The positioning of these three touchpoints, the moments where a user directly interacts with a platform, in the user's journey map (Figure 1) illustrates the sequence of the user's step-by-step interaction with the platform through these three key points of interaction.

User Profile. The User Profile would be an addition to a users' Google Account's existing 'Personal information' page - attaching this to Google's profile makes sense in the context of the features being delivered as a Google Chrome extension-and link to their Just Eat account. Here, as shown in Figure 2 , the users would specify their personal requirements in relation to: dietary preferences, for example vegan; any allergens they should avoid; cultural or personal values, for example halal; foods disliked; and the ability to set consumption targets, for example limiting the number of weekly purchases for either health or financial reasons.

Outlet Selection Page. These are proposed modifications at the outlet selection page, which presents a list of outlets filtered by proximity to the user's specified postcode (ZIP code). As illustrated in Figure 3, this included augmenting each outlet's listing with: its hygiene rating; a personalised outlet recommendation metric based on the User Profile; keywords from outlet reviews and nutritional information, with both metrics having the associated ability to filter by value; and quick reviews, which would highlight keywords associated to a given outlet that were applicable to the user based on their User Profile. The page would also be augmented with goal setting feedback, to set limits and track their takeaway food consumption.

Outlet Menu Page. On a given outlet's menu page, a user can choose their meal items from the menu and choose to see more details about the selected outlet. As shown in Figure 4, the proposed feature augmentations could: block items not suited to the user's profile; provide real-time feedback on the nutritional information of a food item and suggest alternative options; and highlight pertinent reviews of the selected outlet by selecting meta-tagssimilar to existing functionality for review filtering seen on websites such as TripAdvisor. The proposed feature augmentations are shown in red in the user's journey map in Figure 1, providing an overview of the user's augmented experience while interacting with the service platform.

\section{DISCUSSION}

\subsection{The Role of Food Ordering Platforms to Support Healthier Choices and Human-Centred Considerations}

Our design of a web augmentation template for Just Eat highlights clear modifications that could be made to the platform design to support users in making healthy, personal value-led food choices. Though our insights predate the pandemic, they are timely given COVID-19 has significantly accelerated the role that online food ordering platforms play in our food purchasing routines (Shakespeare, 2020) and diets. Our design prominently positions user-desired information at critical point of decision-making in relation to food choice. For example, the provision of an outlet's hygiene rating enables direct comparison of two or more outlets with a comparative cuisine offering. In addition to the provision of supplementary information, users could 


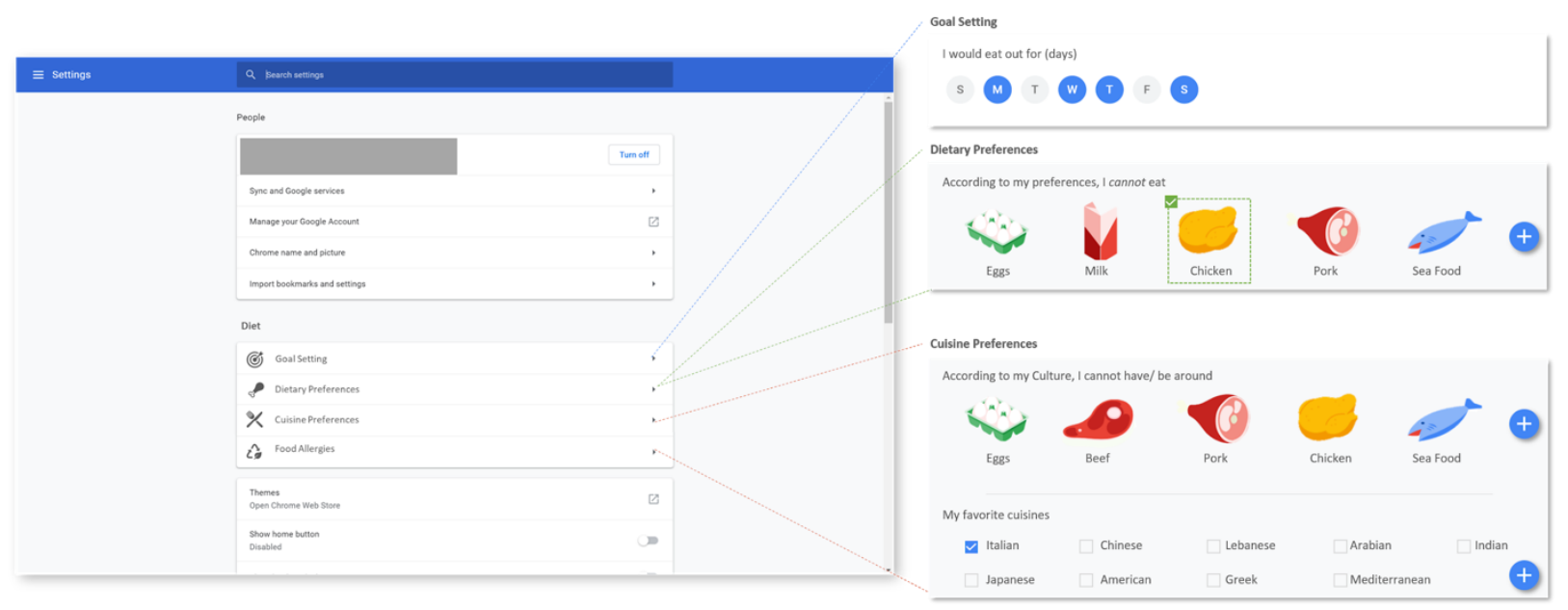

Figure 2: User profile: proposed personal dietary preference settings

enable filtering features that would obscure certain food choices from their view and set consumption goals, enabling them to track their progress. Such additions could potentially dampen the more instinctive and impulsive behavioural aspects of takeaway food ordering ('Smells good, I want it'), and support more deliberate and considered thinking and food choices ('Does it contain too much salt?') (Evans and Stanovich, 2013). Future implementation through web augmentation tools would be required to evaluate the value of the proposed features to Just Eat users and any behavioural impact. As the ideas originated from our user workshops and are supported by prior empirical studies on meal choice heuristics (Scheibehenne, Miesler and Todd, 2007), there is sufficient formative evidence to suggest they have the potential to constructively disrupt the established user journey on the Just Eat platform.

\subsection{Pathway to Impact}

Food ordering platforms are regularly used by millions of people to buy meals. In 2018, Just Eat reported almost a quarter of the UK population purchasing food from the platform (Just Eat plc, 2019a; Park, 2019), and have shown a substantial upward trajectory since 2014 (Just Eat plc, 2015). Given the accepted concept of ubiquitous computing and the rise of dark kitchens (outlets that prepare food solely for food delivery services accessed through online food ordering platforms (Butler, 2017)), computers and smart devices will most likely be our primary point of food selection for takeaway food in the near future. Therefore, the earlier we understand how these platforms influence our choices the sooner we can put in place measures that are supportive of healthier behaviours and reduce the substantial costs to healthcare and the wider economy (McPherson, Marsh and Brown, 2007). Our design provocation is a first step. It makes visible (Klein, 2000) the profit-optimised system design of Just Eat's platform, which in itself could increase agency with regards to their food choice. It provides a clear illustration that is comprehensible beyond an academic audience of what more could be done by online food ordering platforms to empower users to make healthy choices, stimulating the question "why are they not doing this?" However, as a design exercise we must suitably situate how it could lead to a population impact with regards to our dietary habits.

Public health research is concerned with populationlevel health and the translation of science into action. We see two pathways to potential public health impact stemming from our web augmentation template: (i) the ability to model and measure platform behaviour for the purposes of policy development, and (ii) to support advocates and media reporting to exemplify how platforms could be more human-centred. The first step along either pathway is the development of a browser extension. Browser plug-ins such as Takeaway Hygiene Ratings UK (Richard Hodgson, 2017) demonstrate that adding existing supplementary information such as hygiene rating using web augmentation is technologically viable. Other aspects, such as nutritional profiling, are currently not possible due to a lack of data provided by the platform and the outlets, however we have identified a feasible method to create objective outlet-level healthiness indicators through automated content analysis of available online menu text (Goffe et al., 2020). While browser-side augmentation is not novel, its use in public health research is. For the first proposed pathway, such tools must go beyond provision of extra functionality and be effective and GDPR-compliant data collection tools. This would convert a commercial platform into an experimental environment to understand user behaviour. Ensuring that recruitment to future studies are appropriately stratified, they would be able to evaluate different site and extension cues and functionality to identify and understand the mechanisms that promote healthier purchases across different socio-economic and demographic groups. 


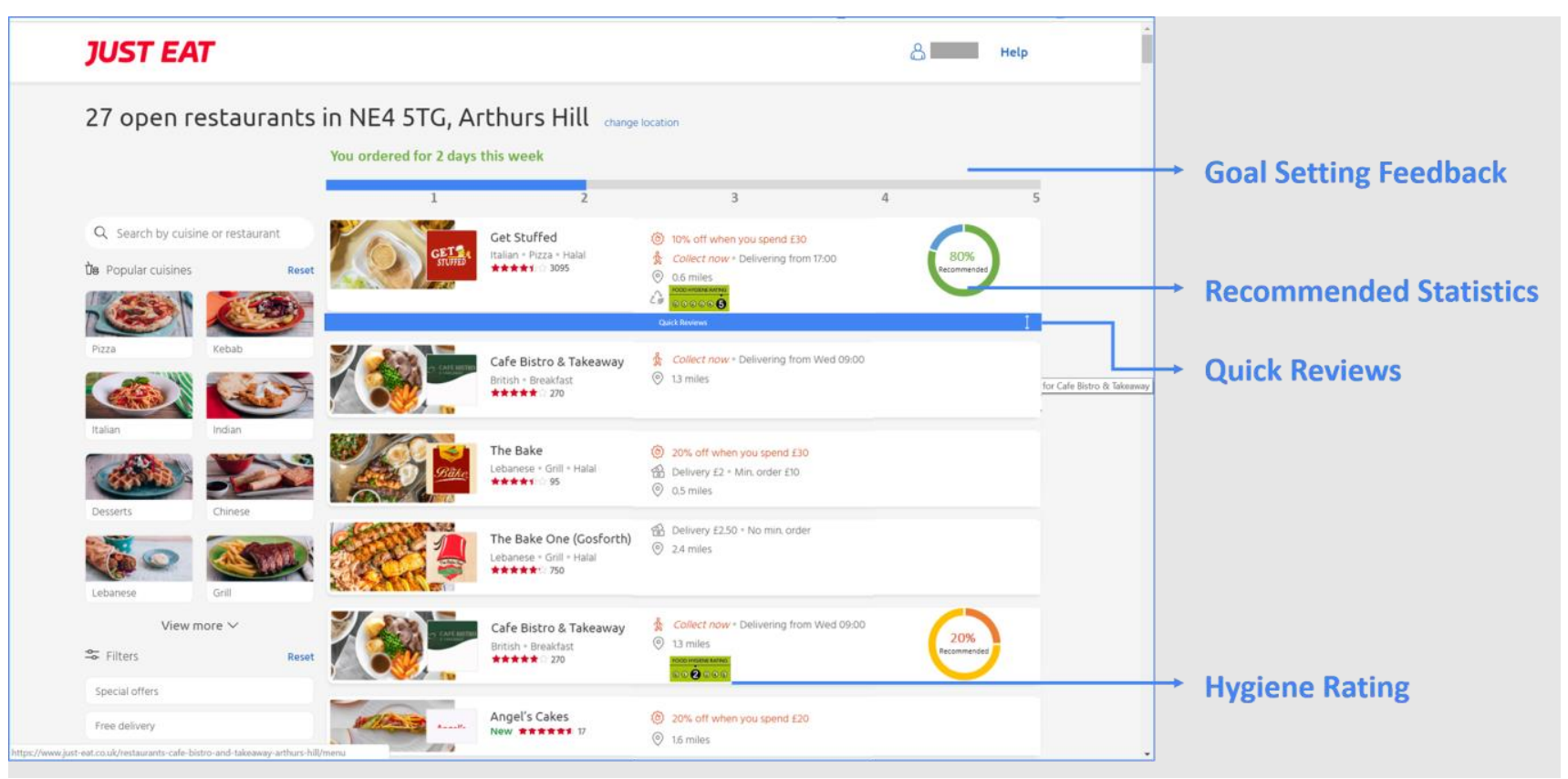

Figure 3: Just Eat's outlet selection page: additional information presented to users that is pertinent to their outlet choice

Researchers and policymakers would be able to trial and simulate the impact on behaviour of considered policies that targeted changes information provision such as meal calorie labelling (Department of Health and Social Care, 2018).

The second pathway using the extension would provide advocates, reporters, and action researchers, with an operational tool that shows how Just Eat, as an exemplar platform, could be used to support improved health and well-being. This could stimulate further ideas and generate similar media reports that may well have played a role in Just Eat's recent notable but low-key announcement of its decision to inconspicuously display an outlet's hygiene rating (Just Eat plc, 2019b). If either pathway is successful, this would have clear implications and applications to other digital platforms that have notable public health issues, such as gambling (Wardle et al., 2019), social media (Seabrook, Kern and Rickard, 2016), and gaming (Király et al., 2014).

\subsection{Human-Centred Design Features for Online Food Ordering}

Our work also highlights what the important design factors are in satisfying people's desires for healthy eating support tools. We have identified three key human-centred design features that would empower food platform users to support their health goals: transparency, personalisation, and self-monitoring:

Transparency relates to making information available, meaningful, and easily accessible to the users. People need more food and outlet information in context to inform their ordering decisions. Just Eat's lack of transparency includes using metrics other than outlet hygiene or food healthiness to order outlet listings, hiding bad user reviews and poor hygiene ratings from users and not incentivising or supporting food outlets to present ingredient, nutrition, or allergen information (see for example the Food Safety Authority of Ireland's MenuCal (Food Safety Authority of Ireland, 2019)). This lack of transparency is 'undermining trust in food' (Crawford and Benjamin, 2019).

Such transparency would enable users to judge not only whether a food option is objectively good but whether it is suitable for them specifically. Personalisation features would allow users to tailor the food ordering experience to their specific values. If a user were able to select a personal nutritional meta-tag such as 'low-fat' which would filter out or label high fat menu items, they would be able to judge which choices would meet their health needs, a particularly useful capability for users with dietary preferences or food allergies. As well as increasing nutritional comprehension, providing a better user experience and a better meal, this could improve data literacy by allowing the user to use not just read health metrics. Enabling users to reflect more deeply on their choices from a better-informed position would enable them to adopt healthier habits, which could be supported through the introduction of selfmonitoring features - the ability for the user to track and regulate platform use to avoid excessive takeaway ordering. This could be as simple as setting a monthly target limit on takeaway orders. Such an approach has already been applied in other areas such as smartphone screen time (Zimmermann, 2021) and online gambling (where it has been shown to have a lasting positive effect on well-being (Auer, Hopfgartner and Griffiths, 2020)). A human-centred platform would support these 


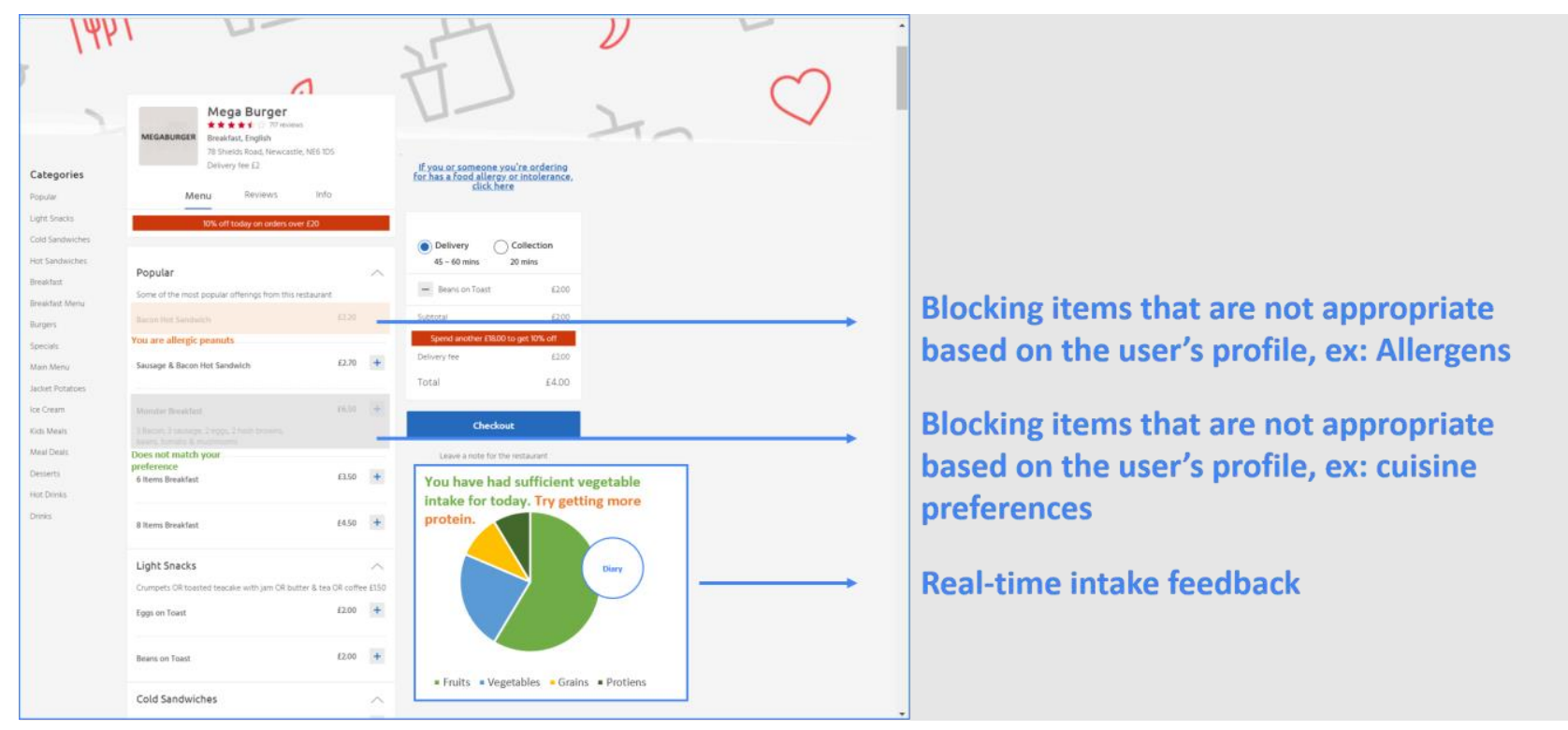

Figure 4: Just Eat's outlet menu page: personalising menu item listing in accordance with user's dietary preferences

healthy human values; it would respect its users by showing them all pertinent information during ordering; it would be considerate of and provide tools to avoid potential harm, even where that mean a slight loss of profit. However offered, such tools could increase agency, control, and satisfaction. If provided by the platform itself, this could increase the user's trust in the brand. The proposed design guidelines can act as design heuristics to build new services or augment existing platforms and as evaluation criteria that could be applied to other lifestyle platforms to explore their potential to improve well-being and support healthy behaviours.

\subsection{Limitations and Future Work}

The formative nature of this research meant that the concepts and ideals were from a limited sample size, reflecting our goals of generative engagement in the spirit of adversarial design instead of seeking generalizability. While our participants were predominately from the age group that is known to be the highest consumers of takeaway food, they did not reflect the views of all members of society who consume takeaway food (Adams et al., 2015). Further research is required to implement the proposed web augmentations presented in our study to understand which design modifications would be of value to different socio-demographic and vulnerable groups. It is also important to acknowledge the intention-behaviour gap, the discrepancy in the translation of intention to action (Sniehotta, Scholz and Schwarzer, 2005). We tried to minimise this gap through the use of both food ordering and purchasing as part of S2, but at this stage in the research it is not known whether the proposed design changes would be used in practice.

\section{CONCLUSION}

We created a design provocation in the form of a web augmentation template for a popular online food ordering platform informed by design workshops with potential users. It exposes design elements of Just Eat that could embrace a humancentred perspective on food ordering behaviour, e.g., identifying healthly options and regulating consumption. In this work, we have shown that it is possible to design improvements to an existing food ordering platform rather than having to create and promote a new e-commerce site. Furthermore, we detail the potential pathways to impact using web augmentation technologies to convert a commercial platform into an experimental environment to evaluate behaviour change. This highlights a new mode of delivery for public health improvement research. Interventions such as our proposed feature augmentations, can surface the existing disempowerment being enacted by platforms and model how those platforms could better support users and their ideals. If such feature augmentations are preferred by platform users, this would provide evidence that could be used by health and well-being advocates and policy makers to influence service providers towards platform design that improves user well-being.

\section{ACKNOWLEDGEMENTS}

We would like to thank all participants for their time and personal insight. Thanks to workshop organisers Kaela Disney, Elizabeth Finley and Rhea Manocha (Objective 1) and Emma Simpson (Objective 2) and to Deanna Bell, Andrew Chu, Kevin McDonald and Leo Qu for their expertise in running the design sprint (Objective 3). Research was funded by the EPSRC Digital Economy Research Centre (EP/M023001/1) and the 'Digital civics goes abroad' (Gray et al., 2019) programme. 


\section{REFERENCES}

Adams, J. et al. (2015) 'Frequency and sociodemographic correlates of eating meals out and take-away meals at home: Cross-sectional analysis of the UK national diet and nutrition survey, waves 1-4 (2008-12)', International Journal of Behavioral Nutrition and Physical Activity, 12(1). doi: 10.1186/s12966-015-0210-8.

Andreassen, C. S., Pallesen, S. and Griffiths, M. D. (2017) 'The relationship between addictive use of social media, narcissism, and self-esteem: Findings from a large national survey', Addictive Behaviors, 64, pp. 287-293. doi:

https://doi.org/10.1016/j.addbeh.2016.03.006.

Anon (2019) 'Just Eat to show food hygiene ratings for all 30,000 restaurants on the app', The Sun. Available at: https://www.thesun.co.uk/money/9512165/just-eatfood-hygiene-ratings-all-restaurants-app/.

Auer, M., Hopfgartner, N. and Griffiths, M. D. (2020) 'The effects of voluntary deposit limit-setting on long-term online gambling expenditure',

Cyberpsychology, Behavior, and Social

Networking. Mary Ann Liebert, Inc., publishers 140 Huguenot Street, 3rd Floor New ..., 23(2), pp. 113-118.

Bleich, S. N. et al. (2017) 'A Systematic Review of Calorie Labeling and Modified Calorie Labeling Interventions: Impact on Consumer and Restaurant Behavior', Obesity, 25(12), pp. 2018-2044. doi: 10.1002/oby.21940.

Buchanan, R. (2001) 'Human dignity and human rights: Thoughts on the principles of humancentered design', Design issues. MIT Press, 17(3), pp. 35-39.

Burgoine, T. et al. (2014) 'Associations between exposure to takeaway food outlets, takeaway food consumption, and body weight in Cambridgeshire, UK: population based, cross sectional study', BMJ. BMJ Publishing Group Ltd, 348. doi: 10.1136/bmj.g1464.

Butler, S. (2017) 'How Deliveroo's "dark kitchens" are catering from car parks', The Guardian.

Available at:

https://www.theguardian.com/business/2017/oct/28 /deliveroo-dark-kitchens-pop-up-feeding-the-citylondon.

Carmichael, J. (2014) 'Google Knows You Better Than You Know Yourself', The Atlantic. Available at:

https://www.theatlantic.com/technology/archive/201 4/08/google-knows-you-better-than-you-knowyourself/378608/.

Chakraborty, A. et al. (2016) 'Stop Clickbait: Detecting and preventing clickbaits in online news media', in 2016 IEEE/ACM International Conference on Advances in Social Networks Analysis and Mining (ASONAM). IEEE, pp. 9-16. doi: 10.1109/ASONAM.2016.7752207.

Chamberlain, S., Sharp, H. and Maiden, N. (2006) 'Towards a framework for integrating agile development and user-centred design', in International Conference on Extreme Programming and Agile Processes in Software Engineering, pp. 143-153.

Clement, J. (2019a) Google's revenue worldwide from 2002 to 2018 (in billion U.S. dollars). Available at:

https://www.statista.com/statistics/266206/googlesannual-global-revenue/.

Clement, J. (2019b) Number of monthly active Facebook users worldwide as of 2nd quarter 2019 (in millions). Available at:

https://www.statista.com/statistics/264810/numberof-monthly-active-facebook-users-worldwide/.

Crawford, A. (2018) 'Just Eat listings include takeaways given zero ratings for hygiene', $B B C$ News. Available at: https://www.bbc.co.uk/news/uk45888709.

Crawford, A. and Benjamin, A. (2019) 'Trust "undermined" by food delivery firms over hygiene', $B B C$ News. Available at: https://www.bbc.co.uk/news/uk-48705066.

Department of Health and Social Care (2018) 'Consultation call: Calorie labelling for food and drink served outside of the home'. Available at: https://www.gov.uk/government/consultations/calori e-labelling-for-food-and-drink-served-outside-ofthe-home.

Díaz, O. (2012) 'Understanding Web Augmentation', in, pp. 79-80. doi: 10.1007/978-3642-35623-0_8.

Díaz, O. et al. (2014) 'End-User Browser-Side Modification of Web Pages', Lecture Notes in Computer Science (including subseries Lecture Notes in Artificial Intelligence and Lecture Notes in Bioinformatics), 8786, pp. 293-307. doi: 10.1007/978-3-319-11749-2_23.

Díaz, O. and Arellano, C. (2015) 'The Augmented Web: Rationales, Opportunities, and Challenges on Browser-Side Transcoding', ACM Trans. Web. New York, NY, USA: ACM, 9(2), pp. 8:1----8:30. doi: 10.1145/2735633.

Díaz, O., Arellano, C. and Azanza, M. (2013) 'A Language for End-user Web Augmentation: Caring for Producers and Consumers Alike', ACM Trans. Web, 7(2), pp. 9:1----9:51. doi: $10.1145 / 2460383.2460388$.

DiSalvo, C. (2012) Adversarial Design. The MIT 
Press.

Dunbar, R. I. M. (2017) 'Breaking Bread: the Functions of Social Eating', Adaptive Human Behavior and Physiology, 3(3), pp. 198-211. doi: 10.1007/s40750-017-0061-4.

Ennals, R., Trushkowsky, B. and Agosta, J. M. (2010) 'Highlighting disputed claims on the web', in Proceedings of the 19th international conference on World wide web - WWW'10. New York, New York, USA: ACM Press, p. 341. doi: 10.1145/1772690.1772726.

Evans, J. S. B. T. and Stanovich, K. E. (2013) 'Dual-Process Theories of Higher Cognition: Advancing the Debate', Perspectives on Psychological Science, 8(3), pp. 223-241. doi: $10.1177 / 1745691612460685$.

Food Safety Authority of Ireland (2019) 'MenuCal helps you put allergens and calories on your menu'. Available at: https://menucal.fsai.ie.

Food Standards Agency (2018) 'Food Hygiene Rating Scheme'. Available at:

https://www.food.gov.uk/safety-hygiene/foodhygiene-rating-scheme.

Fraser, L. K. et al. (2010) 'The Geography of Fast Food Outlets: A Review', International Journal of Environmental Research and Public Health, 7(5), pp. 2290-2308. doi: 10.3390/ijerph7052290.

Goffe, L. et al. (2017) 'Relationship between mean daily energy intake and frequency of consumption of out-of-home meals in the UK National Diet and Nutrition Survey', International Journal of Behavioral Nutrition and Physical Activity, 14(1). doi: 10.1186/s12966-017-0589-5.

Goffe, L. et al. (2018) 'The challenges of interventions to promote healthier food in independent takeaways in England: Qualitative study of intervention deliverers' views', BMC Public Health, 18(1). doi: 10.1186/s12889-018-5096-3.

Hartson, R. and Pyla, P. S. (2012) The UX Book: Process and guidelines for ensuring a quality user experience. Elsevier.

Hillier-Brown, F. C. et al. (2017) 'A description of interventions promoting healthier ready-to-eat meals (to eat in, to take away, or to be delivered) sold by specific food outlets in England: a systematic mapping and evidence synthesis', BMC Public Health, 17(1), p. 93. doi: 10.1186/s12889016-3980-2.

Jaworowska, A. et al. (2014) 'Nutritional composition of takeaway food in the UK', Nutrition \{|\&\} Food Science. Emerald Group Publishing Limited, 44(5), pp. 414-430.

Just Eat plc (2015) Full Year Results. Available at: https://www.justeatplc.com/investors/results- reports.

Just Eat plc (2019a) '2018 Full year Results', Press release. Available at:

https://www.justeatplc.com/news-and-media/pressreleases/2018-full-year-results-announcement.

Just Eat plc (2019b) 'Just Eat starts displaying official food hygiene ratings of all UK restaurants', Press release. Available at:

https://www.justeatplc.com/news-and-media/pressreleases/just-eat-starts-displaying-official-foodhygiene-ratings-all-uk-restaurants.

Just Eat plc (2019c) 'Our technology'. Available at: https://www.justeatplc.com/about-us/ourtechnology.

Just Eat plc (2019d) 'Results and reports'. Available at:

https://www.justeatplc.com/investors/resultsreports.

Just Eat plc (2020) 'Join the UK's leading food delivery provider'. Available at: https://restaurants.just-eat.co.uk/.

Király, O. et al. (2014) 'Chapter 4 - Problematic Online Gaming', in Rosenberg, K. P. and Feder, L. C. (eds) Behavioral Addictions. San Diego: Academic Press, pp. 61-97. doi: https://doi.org/10.1016/B978-0-12-407724-9.000045.

Klein, N. (2000) No Logo. Flamingo, London.

Lambton-Howard, D. et al. (2019) 'WhatFutures: Designing Large-Scale Engagements on WhatsApp', in Proceedings of the $2019 \mathrm{CHI}$ Conference on Human Factors in Computing Systems. New York, NY, USA: ACM (CHI '19), pp. 159:1----159:14. doi: 10.1145/3290605.3300389.

Maccoll, I. and Chalmers, M. (2003) Seamful and seamless design in ubiquitous computing $A$ Population Approach to Ubicomp System Design View project Information Environments Program View project Seamful and Seamless Design in Ubiquitous Computing. Available at: www.equator.ac.uk.

McCabe, S. and Erdem, S. (2021) 'The influence of mortality reminders on cultural in-group versus outgroup takeaway food safety perceptions during the COVID-19 pandemic', Journal of Applied Social Psychology, (December 2020), pp. 363-369. doi: 10.1111/jasp.12740.

McFarlane, N. (2005) 'Fixing web sites with Greasemonkey', Linux Journal. Belltown Media, 2005(138), p. 1.

McPherson, K., Marsh, T. and Brown, M. (2007) Tackling obesities: future choices: Modelling future trends in obesity and the impact on health. Citeseer. 
Miura, K., Giskes, K. and Turrell, G. (2012) 'Socioeconomic differences in takeaway food consumption among adults', Public Health Nutrition. Cambridge University Press, 15(2), pp. 218-226. doi: 10.1017/S136898001100139X.

Park, N. (2019) Population estimates for the UK, England and Wales, Scotland and Northern Ireland: mid-2018. Available at:

https://www.ons.gov.uk/peoplepopulationandcomm unity/populationandmigration/populationestimates/b ulletins/annualmidyearpopulationestimates/mid201 8\%7B\%5C\#\%7Dageing-number-of-over-65scontinues-to-increase-faster-than-the-rest-of-thepopulation.

Parker, G. G., Van Alstyne, M. W. and Choudary, S. P. (2016) Platform revolution: how networked markets are transforming the economyand how to make them work for you. WW Norton I\& Company.

Passport (2018) 'Fast food in the United Kingdom'. Euromonitor International. Available at: https://www.euromonitor.com/.

Prentice, A. M. and Jebb, S. A. (2003) 'Fast foods, energy density and obesity: a possible mechanistic link', Obesity Reviews, 4(4), pp. 187-194. doi: 10.1046/j.1467-789X.2003.00117.x.

Public Health England (2018) Fast food outlets: density by local authority in England. Available at: https://www.gov.uk/government/publications/fastfood-outlets-density-by-local-authority-in-england.

Pyshkin, E. et al. (2016) 'Striving with online addiction with a self-control chrome extension', in 2016 IEEE Symposium Series on Computational Intelligence (SSCI). IEEE, pp. 1-4. doi: 10.1109/SSCI.2016.7850190.

Richard Hodgson (2017) 'Takeaway Hygiene Ratings UK'. Available at:

https://chrome.google.com/webstore/detail/takeawa y-hygiene-

ratings/bkmnhmkibfcgcddfkgmgnecchilhbgmi.

Scheibehenne, B., Miesler, L. and Todd, P. M. (2007) 'Fast and frugal food choices: Uncovering individual decision heuristics', Appetite, 49(3), pp. 578-589. doi: https://doi.org/10.1016/j.appet.2007.03.224.

Seabrook, E. M., Kern, M. L. and Rickard, N. S. (2016) 'Social Networking Sites, Depression, and Anxiety: A Systematic Review', JMIR Ment Health, 3(4), p. e50. doi: 10.2196/mental.5842.

Shakespeare, S. (2020) Changing Consumer Landscape: Dining and quick service restaurants, YouGov. Available at: https://yougov.co.uk/topics/consumer/articlesreports/2020/05/13/changing-consumer-landscapedining-and-quick-servi (Accessed: 23 April 2021).
Storni, C. (2014) 'The problem of De-sign as conjuring: Empowerment-in-use and the politics of seams', Proceedings of the 13th Participatory Design Conference on Research Papers - PDC '14. New York, New York, USA: ACM Press, pp. 161170. doi: $10.1145 / 2661435.2661436$.

Strava (2021) 'Strava'. Available at: https://www.strava.com/.

Suliman, N. A. B. and Mammi, H. B. K. (2017) 'Explicit words filtering mechanism on web browser for kids', in 2017 6th ICT International Student Project Conference (ICT-ISPC). IEEE, pp. 1-6. doi: 10.1109/ICT-ISPC.2017.8075322.

Tomitsch, M. et al. (2018) Design. Think. Make. Break. Repeat. A handbook of methods. Bis Publishers.

Wardle, H. et al. (2019) 'Gambling and public health: we need policy action to prevent harm', BMJ. BMJ Publishing Group Ltd, 365. doi: 10.1136/bmj.l1807.

Watanabe, T., Omori, Y. and others (2020) 'Online consumption during the covid-19 crisis: Evidence from Japan', Covid Economics, 38(16), pp. 218252.

Zimmermann, L. (2021) 'Your screen-time app is keeping track: Consumers are happy to monitor but unlikely to reduce smartphone usage'. 